\title{
Uniform Asymptotic Probability for Multi Renewal Risk Model with Strong Subexponential Tailed Claims
}

\author{
Fotis Loukissas \\ Department of Statistics and Actuarial-Financial Mathematics, \\ University of the Aegean, Samos, Greece. \\ E-mail: floukissas@aegean.gr \\ Alex Karagrigoriou \\ Department of Statistics and Actuarial-Financial Mathematics, \\ University of the Aegean, Samos, Greece. \\ Corresponding author: alex.karagrigoriou@ aegean.gr
}

(Received on October 30, 2021; Accepted on January 4, 2022)

\begin{abstract}
In this paper, we study the uniform asymptotic behavior for the ruin probability in a continuous time renewal counting process. For the proposed model, we assume that the financial claims for each extreme event are compensated by a finite number of independent insurance companies. Moreover, it is assumed that the claims of each insurance company is a sequence of random variables the tail distribution of which belongs to the class of subexponential distributions with finite mean. More specifically the objective of this work is the study of the uniform asymptotic behavior of ruin probability within the class of strongly subexponential distributions, a subclass of subexponential distributions, which provides a convenient framework for investigating heavy tailed distributions. The results are established for two types of asymptotic relations, namely for the common ruin probability of insurance companies and also for the ruin probability of at least one insurance company.
\end{abstract}

Keywords- Insurance risk, Heavy tailed distributions, Subexponential tails, Ruin probability, Renewal model, k-th dimensional risk model, Uniform asymptotic theory.

\section{Introduction}

Nowadays, the occurrence of extreme value events has become increasingly frequent. Earthquakes, wildfire and cyclones can be indicatively mentioned. Furthermore, extreme events could happen in complex computer systems and in construction projects.

The consequences of these events are, in many occasions, catastrophic when financial and social aspects are involved. Risk theory and ruin probabilities are considered to be important parts of insurance mathematics. Although in practice Value-at-Risk and Expected Shortfall are typical risk measures, ruin theory retains a key role not only for risk handling and management in actuarial science but also in various other applied probability areas such as queueing theory or mathematical finance (see e.g. Kostyuchenko, 2018). Apparently, there is a need to develop prediction theories and actuarial methods for analyzing the ruin probabilities of such events. In order to develop these theoretical tools, random variables (like the claims in insurance companies) with heavy tailed distributions are applied.

The model studied in this work is that in each extreme phenomenon, there are a number of ruins, compensated by a finite number $\mathrm{k}$, of insurance companies, which are independent from one another. Another hypothesis in our model is that extreme events happen following the renewal 
counting process. Therefore, the renewal counting process $\mathrm{N}(\mathrm{t})$ shows the number of extreme events that happen during a period of time $\mathrm{t}$ with a mean function $\lambda(t)=E(N(t))$ which tends to infinity as $t \rightarrow \infty$.

The asymptotic ruin probability for claims with heavy-tails has attracted the attention of many investigators in recent years. In the case that the integrated tail distribution of $\mathrm{F}$ is subexponential, (Embrechts and Veraverbeke, 1982) established a simple asymptotic relation. Among numerous other results on this field, the interested reader may refer to papers by Veraverbeke (1977), Hao and Tang (2008), Kim and Lee (2008), Toma and Dedu (2014), D’ Amico et al. (2015) \& Jordanova and Stehlik (2018).

It should be noted that not only the asymptotic but also the finite ruin probability has attracted the interest of researchers. We refer the reader to interesting works by Asmussen (2003) and Tang (2004). For further extensions of these results see the papers by Leipus and Siaulys $(2007,2009)$ \& Dermitzakis and Politis (2011).

These days, many results are based on the assumption of dependence between the actual size and the waiting time of claim. Interested readers are referred to Chen and Yuen (2012) and Li (2015, 2016).

Also, in recent years, the asymptotic performance of insurance quantities for bidimensional risk models has been placed in the center of attention of risk theory researchers. Lu and Zhang (2016) provide asymptotic results for the two-dimensional case under specific assumptions on the associated hazard rate with subexponential claims size. Also, in Foss et al. (2017) we find results for bidimensional ruin probability for subexponential claims size in the case the initial capitals xi, $\mathrm{i}=1,2$ tend to infinity. For further results see Liu et al. (2007), Chen et al. (2011), Chen et al. (2013a, 2013b), Psarrakos (2015), among many others.

Our aim in this work is the study of the uniform asymptotic behavior of ruin probability within the class of strongly subexponential distributions (Korshunov, 2002) which constitute a special subclass of subexponential distributions (see Embrechts et al., 1997) known to provide a convenient framework for studying large classes of heavy tailed distributions.

The paper is organized as follows. The notations and a number of preliminary results are presented in Section 2 with the main results provided in Section 3. Some concluding remarks can be found in Section 4.

\section{Notations and Preliminary Results}

Let assume that $k$ is the number of insurance companies having an initial capital $x_{i}, i=1,2,3, \ldots, k$ and a premium $c_{i}, i=1,2, \ldots, k$ each. We symbolize with $X_{i j}, i=1,2,3, \ldots, k, j=1,2,3, \ldots, N(t)$ the random variables that represent the damage of the $i^{\text {th }}$ company during the $j^{\text {th }}$ extreme event.

In this paper, we write the vector claims sizes $\vec{X}_{j}=\left(X_{1 j}, \ldots, X_{k j}\right)^{T}, j=1,2, \ldots, N(t)$ with distribution functions $\left(F_{1}, \ldots, F_{k}\right)^{T}$ and finite means $\left(v_{1}, \ldots, v_{k}\right)^{T}$ where $b^{T}$ represents the transpose of the vector $b$. It is assumed that the claim amounts vectors $\vec{X}_{j}=\left(X_{1 j}, \ldots, X_{k j}\right)^{T}$ are independent identically distributed copies of $\vec{X}=\left(X_{1}, \ldots, X_{k}\right)^{T}$ and are independent of the renewal counting process $\{N(t), t \geq 0\}$. Let us denote by $\left\{\theta_{i}, i \geq 1\right\}$ the independent identically distributed inter-arrival 
times with common finite positive mean $1 / \lambda$.

With the initial surplus vector $\vec{x}=\left(x_{1}, \ldots, x_{k}\right)^{T}$ and the constant premium rate vector denoted by $\vec{c}=\left(c_{1}, \ldots, c_{k}\right)^{T}$, the surplus vector process $\vec{R}(t)=\left(R_{1}(t), \ldots, R_{k}(t)\right)^{T}$ is given by

$\vec{R}(t)=\vec{x}+\vec{c} t-\sum_{j=1}^{N(t)} \vec{X}_{j}, \quad t \geq 0$,

where,

$R_{i}(t)=x_{i}+c_{i} t-\sum_{j=1}^{N(t)} X_{i j}, \quad t \geq 0, \quad i=1, \ldots, k$

represents the surplus of each insurance company. Furthermore, the time of ruin of each insurance company is expressed by

$T_{i}\left(x_{i}\right)=\inf \left\{t>0: R_{i}(t)<0 \mid R_{i}(0)=x_{i}\right\}, \quad i=1, \ldots, k$.

In the literature of the ruin probabilities, we find the following types of ruin time. The first type is defined by

$T_{\text {max }}(\vec{x})=\inf \left\{t>0: \max \left\{R_{1}(t), \ldots, R_{k}(t)\right\}<0 \mid \vec{R}(0)=\vec{x}\right\}$

representing the first time for which all $R_{i}(t), i=1, \ldots, k$ become negative at the same time whereas the second one is

$T_{\min }(\vec{x})=\inf \left\{t>0: \min \left\{R_{1}(t), \ldots, R_{k}(t)\right\}<0 \mid \vec{R}(0)=\vec{x}\right\}$

which represents the $1^{\text {st }}$ time that $R_{1}(t)$ or $R_{2}(t)$ or..., $R_{k}(t)$ became negative. The $3^{\text {rd }}$ type is defined by

$T_{\text {and }}(\vec{x})=\max \left\{T_{1}\left(x_{1}\right), \ldots, T_{k}\left(x_{k}\right)\right\}$

representing the first time for which all $R_{i}(t), i=1, \ldots, k$ become negative but not at the same time and the last type is

$T_{\text {sum }}(\vec{x})=\inf \left\{t>0: R_{1}(t)+\cdots+R_{k}(t)<0 \mid \vec{R}(0)=\vec{x}\right\}$

representing the first time for which the aggregate surplus process of $R_{i}(t), i=1, \ldots, k$ goes below zero.

Then, the probabilities of ruin are given respectively by

$$
\begin{aligned}
& \psi_{\vee}(\vec{x}, t)=P\left(T_{\max }(\vec{x}) \leq t\right) \\
& \psi_{\wedge}(\vec{x}, t)=P\left(T_{\min }(\vec{x}) \leq t\right) \\
& \psi_{\text {and }}(\vec{x}, t)=P\left(T_{\text {and }}(\vec{x}) \leq t\right) \\
& \psi_{\text {sum }}(\vec{x}, t)=P\left(T_{\text {sum }}(\vec{x}) \leq t\right) .
\end{aligned}
$$

Compared to the prosperity of the study on bidimensional risk models, the results on multidimensional risk models have been quite limited. Indeed, recent results mostly on bidimensional models, include large deviations for sums of claim-size vectors (Fu et al., 2021), asymptotic sum-ruin probabilities (Sun et al., 2021a, 2021b) and uniform asymptotics for ruin 
probabilities (Cheng, 2021; Wang et al., 2021). On the other hand, we refer for instance to the work by Li and Yang (2015) who studied a special case of the multidimensional risk model with a Poisson claim number process. They established results associated with the finite time ruin probabilities defined in (2.5) and (2.7). Konstantinides and Li (2016) established a continuous time model with a constant force of interest and derived a precise asymptotic expansion for the finite time ruin probability defined in (2.6) while $\mathrm{Li}$ (2016) studied precise asymptotic results for the finite time ruin probabilities defined in (2.5) and (2.6) in a multidimensional risk model with a stochastic return driven by a geometric Levy process. Finally, bounds for multidimensional ruin probabilities in regime-switching models have been recently proposed (Gajek and Rudź, 2021).

Last but not least we refer to Cheng and Yu (2019) obtained uniform asymptotic results with strongly subexponential claims in a multidimensional risk model defined in (2.7). In this work we concentrate on the two types of ruin probabilities defined in (2.4) and (2.5). It should be noted that the present work contributes to the existing literature and complements the ruin asymptotic theory by providing asymptotic results for the case of ruin time Tmax, given in (2.4) which to the best of our knowledge, has not been considered before and it is the most interesting for the actuarial science while at the same time, investigates the case Tmin given in (2.5) under the general framework of the multi renewal risk model within the class of strongly subexponential distributions.

\subsection{Preliminary Results}

In order to secure that the certainty of ruin in each occurrence is non existed, we proceed with assuming that the safety loading conditions hold, namely $\mu_{i}=c_{i} / \lambda-v_{i}>0, i=1,2, \ldots, k$.

In the rest of the paper we use the following notations. Consider positive functions $a(\cdot)$ and $b(\cdot)$. The notation $a(x) \lesssim b(x)$ is used when limsup $a(x) / b(x) \leq 1$ while the notation $a(x) \gtrsim b(x)$ is used when liminf $a(x) / b(x) \geq 1$ for $x \rightarrow \infty$. Finally, in case the limit lim $a(x) / b(x)=1$ holds then the notation $a(x) \sim b(x)$ is used.

As indicated in the Introduction our aim in this work is the study of the uniform asymptotic behavior of ruin probability within $S$ which represents the class of subexponential distributions (see Embrechts et al., 1997). Assume that the support of the df $F$ is on $[0, \infty)$ and $\bar{F}=1-F$. Then, $F \in$ $\mathrm{S}$ if it satisfies the expression

$$
\lim _{x \rightarrow \infty} \frac{\overline{F^{* n}}(x)}{\bar{F}(x)}=n, \quad \forall n \geq 2,
$$

where, $F^{* n}$ represents the $n^{\text {th }}$ convolution of $F$. We further focus on the subclass $\mathrm{S}_{*}$ of strongly subexponential distributions proposed by Korshunov (2002). Consider a subexpontential distribution function $F$ with finite stop-loss premiums, namely

$$
\int_{0}^{\infty} F(y) d y<\infty .
$$

For any $u>0$ we can state the following

$$
\bar{F}_{u}(x)=\int_{x}^{x+u} \bar{F}(y) d y
$$

Then, $F$ is defined to be strongly subexponential, provided that the following relation holds uniformly for $u \in[1, \infty)$ : 


$$
\overline{F_{u}^{* 2}}(x) \sim 2 \overline{F_{u}}(x)
$$

Finally, note that the following proposition holds (see Korshunov, 2002) that is useful for our main aim.

Proposition 1 If $F \in S_{*}$ with finite mean $v<0$, then the following holds uniformly for $n \geq 1$ :

$P\left(\max _{1 \leq \kappa \leq n} S_{k}>x\right) \sim \frac{1}{|v|} \int_{x}^{x+n|v|} \bar{F}(y) d y, \quad x \rightarrow \infty$

where, $S_{k}=\sum_{i=1}^{k} X_{i}$ represents the partial sum.

Recall that $\mathrm{S}$ is a subclass of $L$, the family of long tail distributions. It is well known that $F \in L$ with support on $[0, \infty)$ if:

$$
\lim _{x \rightarrow \infty} \frac{\bar{F}(x-y)}{\bar{F}(x)}=1, \quad \forall y>0
$$

Furthermore, note that for any $F \in \mathrm{L}$ there exists an increasing non-negative function $l(x)=o(x)$ with $l(x) \rightarrow \infty$ as $x \rightarrow \infty$ such that $\bar{F}(x \pm l(x)) \sim \bar{F}(x), \quad x \rightarrow \infty$.

The expressions $l(x)=o(x / \ln x)$ and $l(x)=o\left(x^{1-\alpha}\right), \alpha \in(0,1)$, are two representative examples associated with the lognormal and Weibull distributions, respectively. As for the case of intermediate regularly varying distributions (more specifically, regularly varying distributions) the function $l(x)=x^{1 / 2}$ can be used. More information about the increasing function $l(x)$ can be found find in Foss et al. (2011).

The result below, which will be used later, has been obtained by Tang et al. (2001).

Lemma 1 For the renewal counting process $N(t)$ with $k \geq 1$ it holds that $\lim \sum_{n>(1+\epsilon) \lambda t} n_{t \rightarrow \infty}^{k} P(N(t) \geq n)=0$.

The uniform asymptotic behavior for two function $a(x)$ and $b(x)$ which we study in this paper has the following formula

$$
\lim _{t \rightarrow \infty} \sup _{f(x) \geq t} \frac{a(x)}{b(x)}=1
$$

where, $f(\cdot)$ is an increasing function.

Now, we prove the following relation which is useful in the main result.

Lemma 2 Let $\left\{X_{k}, k \geq 1\right\}$ be a non-negative independent and identically distributed sequence of random variables with common d.f. $F \in S_{*}$. Then holds,

$P\left(\sum_{i=1}^{n} X_{i}>x\right) \succsim n \bar{F}(x)$,

as $n \rightarrow \infty$ uniformly for $l(x) \geq n$.

Proof. From the Bonferroni inequality we get 


$$
\begin{aligned}
& P\left(\sum_{i=1}^{n} X_{i}>x\right) \geq P\left(\sum_{i=1}^{n} X_{i}>x, \max _{1 \leq 1 \leq n} X_{i}\right) \\
& \geq \sum_{i=1}^{n} P\left(\sum_{i=1}^{n} X_{i}>x, X_{i}>x\right)-\sum_{1 \leq k \leq l \leq n} P\left(\sum_{i=1}^{n} X_{i}>x, X_{k}>x, X_{l}>x\right) \\
& \geq \sum_{i=1}^{n} P\left(X_{i}>x\right)-\left(n P\left(X_{i}>x\right)\right)^{2} \geq n \bar{F}(x)(1-n \bar{F}(x)) .
\end{aligned}
$$

For a sequence $\left\{X_{k}, k \geq 1\right\}$ of non-negative random variables with $\mathrm{df} F$ and finite mean holds $n F(x) \rightarrow 0$ as $n \rightarrow \infty$ uniformly for $l(x) \geq n$. So, we conclude

$$
\lim _{t \rightarrow \infty} \sup _{l(x) \geq n} \frac{P\left(\sum_{i=1}^{n} X_{i}>x\right)}{n \bar{F}(x)} \geq 1 .
$$

Finally, the following inequality will be used in proving the main result in the next section:

Let $a, b$ and $c$ be constants satisfying the inequality $a<b<c$ and let $f(y)$ be a non-increasing function defined in $[a, c]$. Then, it holds that

$\int_{a}^{c} f(y) d y \leq \frac{c-a}{b-a} \int_{a}^{b} f(y) d y$.

\section{Main Results}

In this work, we are interested in carrying out the results for two uniform asymptotic relations for ruin probabilities in the framework of the multi-dimensional renewal risk model. The first relation is in regard to $\psi_{\mathrm{v}}(\vec{x}, t)$ and the second one is in relation to $\psi_{\wedge}(\vec{x}, t)$ given in expressions (2.4) and (2.5) respectively.

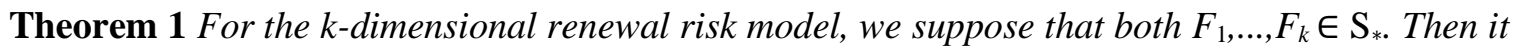
holds

$\psi_{\mathrm{v}}(\vec{x}, t) \sim(\lambda t)^{k} \prod_{i=1}^{k} \bar{F}_{i}\left(x_{i}\right)$

as $t \rightarrow \infty$, uniformly for $l_{i}\left(x_{i}\right) \geq \lambda c_{i} t^{2}, i=1,2, \ldots, k$.

\section{Proof}

We first proceed with the proof of the asymptotic lower bound

$$
\begin{aligned}
& \lim _{t \rightarrow \infty} \sup _{l_{i}\left(x_{i}\right) \geq \lambda c_{i} t^{2}} \frac{\psi_{\mathrm{v}}(\vec{x}, t)}{(\lambda t)^{k} \prod_{i=1}^{k} \bar{F}_{i}\left(x_{i}\right)} \geq 1, \quad i=1,2, \ldots, k . \\
& \psi_{\mathrm{v}}(\vec{x}, t)=P\left(T_{\max }(\vec{x}) \leq t\right)=P\left(\bigcap_{i=1}^{k}\left\{\sum_{j=1}^{N(t)}\left(X_{i j}-c_{i} t\right)>x_{i}\right\}\right) \\
& =\sum_{n=0}^{\infty} P\left(\bigcap_{i=1}^{k}\left\{\sum_{j=1}^{n} X_{i j}>x_{i}+n c_{i} t\right\}, N(t)=n\right) \\
& \geq \sum_{(1-\epsilon) \lambda t \leq n \leq(1+\epsilon) \lambda t} P\left(\bigcap_{i=1}^{k}\left\{\sum_{j=1}^{n} X_{i j}>x_{i}+n c_{i} t\right\}, N(t)=n\right)
\end{aligned}
$$


$\geq P\left(\bigcap_{i=1}^{k}\left\{\sum_{j=1}^{(1-\epsilon) \lambda t} X_{i j}>x_{i}+(1+\epsilon) \lambda c_{i} t^{2}\right\},(1-\epsilon) \lambda t \leq N(t) \leq(1+\epsilon) \lambda t\right)$

$\geq P\left(\bigcap_{i=1}^{k}\left\{\sum_{j=1}^{(1-\epsilon) \lambda t} X_{i j}>x_{i}+(1+\epsilon) \lambda c_{i} t^{2}\right\}\right) P\left(\left|\frac{N(t)}{\lambda t}-1\right| \leq \epsilon\right)$.

Observe that the following well-known result holds for the renewal counting process $\mathrm{N}(\mathrm{t})$ :

$N(t) / \lambda(t) \rightarrow_{p} 1, \quad \lambda(t) \sim \lambda$ tas $t \rightarrow \infty$

or equivalently

$\lim _{t \rightarrow \infty} P\left(\left|\frac{N(t)}{\lambda t}-1\right| \leq \epsilon\right)=1$

for any arbitrary $\epsilon \in(0,1)$.

As $X_{1 i}, X_{2 i}, \ldots, X_{k j}$ are independent with distribution $F_{1}, F_{2}, \ldots, F_{k} \in L$ and if we take $\epsilon \rightarrow 0$ combining with Lemma (2) we come to the following inequality

$\psi_{\mathrm{v}}(\vec{x}, t) \gtrsim \prod_{i=1}^{k} \lambda t \bar{F}_{i}\left(x_{i}+\lambda c_{i} t^{2}\right) \gtrsim \prod_{i=1}^{k} \lambda t \bar{F}_{i}\left(x_{i}+l_{i}(x)\right) \sim \prod_{i=1}^{k} \lambda t \bar{F}_{i}\left(x_{i}\right)$,

as $t \rightarrow \infty$, uniformly for $l_{i}\left(x_{i}\right) \geq \lambda c_{i} t^{2}, i=1,2, \ldots, k$. Taking $\epsilon \rightarrow 0$ we come to the following asymptotic inequality

$\lim _{t \rightarrow \infty} \sup _{l_{i}\left(x_{i}\right) \geq \lambda c_{i} t^{2}} \frac{\psi_{\mathrm{v}}(\vec{x}, t)}{(\lambda t)^{k} \prod_{i=1}^{k} \bar{F}_{i}\left(x_{i}\right)} \geq 1, \quad i=1,2, \ldots, k$.

Now, we establish the asymptotic relation for the upper bound, namely

$\lim _{t \rightarrow \infty} \sup _{l_{i}\left(x_{i}\right) \geq \lambda c_{i} t^{2}} \frac{\psi_{\mathrm{v}}(\vec{x}, t)}{(\lambda t)^{k} \prod_{i=1}^{k} \bar{F}_{i}\left(x_{i}\right)} \leq 1, \quad i=1,2, \ldots, k$.

For the proof, we choose an arbitrarily $\epsilon \in(0,1)$ and we define the sequence of random variables as $\tilde{X}_{i j}=X_{i j}-c_{i} \theta_{i}, \quad i=1, \ldots, k$ with finite negative mean $\mu_{i}$. We notice that the increments $\tilde{X}_{i j}$ and the counting process $N(t)$ are not independent anymore, so we continue as follows: We notice that

$$
\begin{aligned}
& \psi_{\mathrm{v}}(\vec{x}, t)=P\left(\bigcap_{i=1}^{k}\left\{\max _{0<m \leq N(t)} \sum_{j=1}^{m}\left(X_{i j}-c_{i} \theta_{i}\right)>x_{i}\right\}\right) \\
& =P\left(\bigcap_{i=1}^{k}\left\{\max _{0<m \leq N(t)} \sum_{j=1}^{m} \tilde{X}_{i j}>x_{i}\right\}\right)=\sum_{n \leq(1+\epsilon) \lambda t} P\left(\bigcap_{i=1}^{k}\left\{\max _{0<m \leq n} \sum_{j=1}^{m} \tilde{X}_{i j}>x_{i}\right\}, N(t)=n\right) \\
& +\sum_{n>(1+\epsilon) \lambda t} P\left(\bigcap_{i=1}^{k}\left\{\max _{0<m \leq n} \sum_{j=1}^{m} \tilde{X}_{i j}>x_{i}\right\}, N(t)=n\right)=J_{1}(\vec{x}, n, t)+J_{2}(\vec{x}, n, t) .
\end{aligned}
$$

For the first term, 


$$
\begin{aligned}
& J_{1}(\vec{x}, n, t)=\sum_{n \leq(1+\epsilon) \lambda t} P\left(\bigcap_{i=1}^{k}\left\{\max _{0<m \leq n} \sum_{j=1}^{m} \tilde{X}_{i j}>x_{i}\right\}, N(t)=n\right) \\
& \leq \sum_{n \leq(1+\epsilon) \lambda t} P\left(\bigcap_{i=1}^{k}\left\{\max _{0<m \leq n} \sum_{j=1}^{m} \tilde{X}_{i j}>x_{i}\right\}\right)
\end{aligned}
$$

and Proposition 1 leads to

$$
\begin{aligned}
& J_{1}(\vec{x}, n, t) \lesssim \sum_{n \leq(1+\epsilon) \lambda t} \prod_{i=1}^{k} \frac{1}{\left|\mu_{i}\right|} \int_{x_{i}}^{x_{i}+n\left|\mu_{i}\right|} P\left(\tilde{X}_{i}>y\right) d y \\
& \leq \prod_{i=1}^{k} \frac{1}{\left|\mu_{i}\right|} \int_{x_{i}}^{x_{i}+(1+\epsilon)\left|\mu_{i}\right| \lambda t} P\left(\tilde{X}_{i}>y\right) d y \leq \prod_{i=1}^{k}(1+\epsilon) \frac{1}{\left|\mu_{i}\right|} \int_{x_{i}}^{x_{i}+\left|\mu_{i}\right| \lambda t} P\left(\tilde{X}_{i}>y\right) d y \\
& \leq \prod_{i=1}^{k}(1+\epsilon)\left(\lambda t \bar{F}_{i}\left(x_{i}\right)\right)
\end{aligned}
$$

where, in the second step we use the inequality (2.12). If we take $\epsilon \rightarrow 0$ then the asymptotic inequality holds

$\frac{J_{1}(\vec{x}, n, t)}{(\lambda t)^{k} \prod_{i=1}^{k} \bar{F}_{i}\left(x_{i}\right)} \lesssim 1$

as $t \rightarrow \infty$, uniformly for $l_{i}\left(x_{i}\right) \geq \lambda c_{i} t^{2}, i=1,2, \ldots, k$.

Turning now to the 2 nd term we can write that

$$
\begin{aligned}
& J_{2}(\vec{x}, n, t)=\sum_{n>(1+\epsilon) \lambda t} P\left(\bigcap_{i=1}^{k}\left\{\max _{0<m \leq n} \sum_{j=1}^{m} \tilde{X}_{i j}>x_{i}\right\}, N(t)=n\right) \\
& \leq \sum_{n>(1+\epsilon) \lambda t} P\left(\bigcap_{i=1}^{k}\left\{\max _{0<m \leq n} \sum_{j=1}^{m} X_{i j}>x_{i}\right\}, N(t)=n\right) .
\end{aligned}
$$

We define the sequence of independent random variables as $X_{i j}^{*}=X_{i j}-v_{i}-1, \quad i=1, \ldots, k$ with $E\left(X_{i j}^{*}\right)=-1$. So,

$J_{2}(\vec{x}, n, t)=\sum_{n>(1+\epsilon) \lambda t} P\left(\bigcap_{i=1}^{k}\left\{\max _{0<m \leq n} \sum_{j=1}^{m} X_{i j}^{*}>x_{i j}-\left(v_{\iota}+1\right)\right\}\right) P(N(t)=n)$.

We use Proposition 1 to reach the following result

$J_{2}(\vec{x}, n, t) \lesssim \sum_{n>(1+\epsilon) \lambda t} \prod_{i=1}^{k} \int_{x_{i}-\left(v_{i}+1\right)}^{x_{i}-\left(v_{i}+1\right)+n} P\left(X_{i}^{*}>y\right) d y P(N(t)=n)$ 
$\leq \sum_{n>(1+\epsilon) \lambda t} \prod_{i=1}^{k}\left(n P\left(X_{i}^{*}>x_{i}-\left(v_{\iota}+1\right)\right)\right) P(N(t)=n)$

$\leq \sum_{n>(1+\epsilon) \lambda t} n^{k} P(N(t)=n) \prod_{i=1}^{k} \bar{F}_{i}\left(x_{i}\right) \leq \sum_{n>(1+\epsilon) \lambda t} n^{k} P(N(t) \geq n)(\lambda t)^{k} \prod_{i=1}^{k} \bar{F}_{i}\left(x_{i}\right)$.

Lemma 1 for $t$ tending to infinity, yields

$J_{2}(\vec{x}, n, t) \sim o\left((\lambda t)^{k} \prod_{i=1}^{k} \bar{F}_{i}\left(x_{i}\right)\right)$,

uniformly for $l_{i}\left(x_{i}\right) \geq \lambda c_{i} t^{2}, i=1,2, \ldots, k$. Combining relations (3.6) and (3.7) we deduce that,

$$
\lim _{t \rightarrow \infty} \sup _{l_{i}\left(x_{i}\right) \geq \lambda c_{i} t^{2}} \frac{\psi_{\mathrm{v}}(\vec{x}, t)}{(\lambda t)^{k} \prod_{i=1}^{k} \bar{F}_{i}\left(x_{i}\right)} \leq 1 \text {. }
$$

The above Theorem for $k=1$ reduces to the result below:

Proposition 2 If the $d f F \in S_{*}$ with finite mean then for the renewal counting process $N(t)$ holds $\psi(x, t)=P(T(x) \leq t) \sim \lambda t \bar{F}(x)$,

as $t \rightarrow \infty$ uniformly for $l(x) \geq \lambda c t^{2}$.

Now, we turn our attention to prove a result for $\psi_{\wedge}(\vec{x}, t)$.

Theorem 2 For the $k$-dimensional renewal risk model, we suppose that $F_{1}, F_{2}, \ldots, F_{k} \in \mathrm{S}_{*}$ with finite means. Then it holds

$\psi_{\wedge}(\vec{x}, t) \sim \lambda t \sum_{i=1}^{k} \bar{F}_{i}\left(x_{i}\right)$

as $t \rightarrow \infty$, uniformly for $l_{i}\left(x_{i}\right) \geq \lambda c_{i} t^{2}, i=1,2, \ldots, k$.

Proof.

The result will be proved by induction. Thus, for $k=2$ using relation (3.8) we get

$\psi_{\wedge}(\vec{x}, t)=P\left(T_{\min }(\vec{x}) \leq t\right)=P\left(T_{1}(\vec{x}) \leq t\right)+P\left(T_{2}(\vec{x}) \leq t\right)-P\left(T_{\max }(\vec{x}) \leq t\right)$

$\sim \lambda t \sum_{i=1}^{2} \bar{F}_{l}\left(x_{i}\right)-P\left(T_{\max }(\vec{x}) \leq t\right)$.

Hence, it suffices to show that

$P\left(T_{\max }(\vec{x}) \leq t\right)=o(1) \lambda t \sum_{i=1}^{2} \bar{F}_{i}\left(x_{i}\right)$

From Theorem 1 we get

$\frac{P\left(T_{\max }(\vec{x}) \leq t\right)}{\lambda t \sum_{i=1}^{2} \bar{F}_{i}\left(x_{i}\right)} \sim \frac{(\lambda t)^{2} \prod_{i=1}^{2} \bar{F}_{i}\left(x_{i}\right)}{\lambda t \sum_{i=1}^{2} \bar{F}_{i}\left(x_{i}\right)}=\frac{1}{\sum_{i=1}^{2} \frac{1}{\lambda t \bar{F}_{i}\left(x_{i}\right)}}=o(1)$

as $t \rightarrow \infty$, uniformly for $l_{i}\left(x_{i}\right) \geq \lambda c_{i} t^{2}, i=1,2$.

Now, let us assume that (3.9) holds true for $k-1$ : 
$\psi_{\wedge}(\vec{x}, t)=P\left(T_{\min }(\vec{x}) \leq t\right) \sim \lambda t \sum_{i=1}^{k-1} \bar{F}_{i}\left(x_{i}\right)$

as $t \rightarrow \infty$, uniformly for $l_{i}\left(x_{i}\right) \geq \lambda c_{i} t^{2}, i=1,2, \ldots, k-1$. Now, we prove that the above relation holds for $k$.

$\psi_{\wedge}(\vec{x}, t)=P\left(T_{\min }(\vec{x}) \leq t\right)=P\left(\bigcup_{i=1}^{k} T_{i}\left(x_{i}\right) \leq t\right)$

$=P\left(\bigcup_{i=1}^{k-1} T_{i}\left(x_{i}\right) \leq t\right)+P\left(T_{k}\left(x_{k}\right) \leq t\right)-P\left(\bigcup_{i=1}^{k-1} T_{i}\left(x_{i}\right) \leq t, T_{k}\left(x_{k}\right) \leq t\right)$

$\sim \lambda t \sum_{i=1}^{k} \bar{F}_{i}\left(x_{i}\right)-P\left(\mathrm{U}_{i=1}^{k-1} T_{i}\left(x_{i}\right) \leq t, T_{k}\left(x_{k}\right) \leq t\right)$.

Using the asymptotic relation (3.12) and Proposition 2, we get

$\frac{P\left(\bigcup_{i=1}^{k-1} T_{i}\left(x_{i}\right) \leq t, T_{k}\left(x_{k}\right) \leq t\right)}{\lambda t \sum_{i=1}^{k} \bar{F}_{i}\left(x_{i}\right)}=\frac{\left(\bigcup_{i=1}^{k-1} T_{i}\left(x_{i}\right) \leq t\right) \cdot P\left(T_{k}\left(x_{k}\right) \leq t\right)}{\lambda t \sum_{i=1}^{k} \bar{F}_{i}\left(x_{i}\right)}$

$\sim \frac{\lambda t \sum_{i=1}^{k-1} \bar{F}_{i}\left(x_{i}\right) \lambda t \bar{F}_{k}\left(x_{k}\right)}{\lambda t \sum_{i=1}^{k} \bar{F}_{i}\left(x_{i}\right)}=\frac{\sum_{i=1}^{k-1} \bar{F}_{i}\left(x_{i}\right) \lambda t \bar{F}_{k}\left(x_{k}\right)}{\sum_{i=1}^{k} \bar{F}_{i}\left(x_{i}\right)}$

$=\frac{1}{\frac{1}{\lambda t \bar{F}_{k}\left(x_{k}\right)}+\frac{1}{\lambda t \sum_{i=1}^{k-1} \bar{F}_{i}\left(x_{i}\right)}}=o(1)$

as $t \rightarrow \infty$, uniformly for $l_{i}\left(x_{i}\right) \geq \lambda c_{i} t^{2}, i=1,2, \ldots, k$.

\section{Conclusions}

In this work we focus and investigate the uniform asymptotic behavior of ruin probability within the class of strongly subexponential distributions which constitute a special subclass of subexponential distributions (see Embrechts et al., 1997) known to provide a convenient framework for studying large classes of heavy tailed distributions. The theoretical results are established for two types of asymptotic relations with the first describing the common ruin probability of insurance companies, while the second referring to the ruin probability of at least one insurance company.

Compared to the prosperity of the study on bidimensional risk models, the results on multidimensional risk models have been quite limited. Indeed, only a handful of works deal with such issues (see e.g. the works of Li with various co-authors ( $\mathrm{Li}$ and Yang, 2015; Konstantinides and $\mathrm{Li}, 2016$; $\mathrm{Li}, 2016$ ). As a result, the present work is filling up the gap with the main contributions lying on the fact that the asymptotic results are characterized by a great generality since they are established for the general multidimensional renewal risk model and at the same time being quite simple without having to rely on any restricted assumptions and in that sense, they provide a significant addition to the relevant literature.

Future research directions include the study of the dependent renewal risk model among claims, the dependence structure between the waiting time for a claim and its actual size, the ruin probabilities in a general economic environment and the probability of randomly weighted sums of independent-dependent random variables. 
Conflict of Interest

The authors confirm that there is no conflict of interest to declare for this publication.

\section{Acknowledgments}

This work was completed as part of the postdoctoral research of the first author and it is part of the activities of the Laboratory of Statistics and Data Analysis of the University of the Aegean. The authors wish to express their appreciation to the anonymous reviewers and the handling Editor for their constructive comments and recommendations.

\section{References}

Asmussen, S. (2003). Applied probability and queues. 2nd edition. Springer-Verlag, New York.

Chen, Y., \& Yuen, K.C. (2012). Precise large deviations of aggregate claims in a size-dependent renewal risk model. Insurance Mathematics and Economics, 51(2), 457-461.

Chen, Y., Yuen, K.C., \& Ng, K.W. (2011). Asymptotics for the ruin probabilities of a two-dimensional renewal risk model with heavy- tailed claims. Applied Stochastic Models in Business and Industry, 27(3), 290-300.

Chen, Y., Wang, L., \& Wang, Y. (2013a). Uniform asymptotics for the finite-tine ruin probability of two kinds of nonstandard bidimensional risk models. Journal of Mathematical Analysis and Applications, 401(1), 114-129.

Chen, Y., Wang, Y., \& Wang, K. (2013b). Asymptotics results for ruin probability of a two-dimensional renewal risk model. Stochastic Analysis and Applications, 31(1), 80-91.

Cheng, D. (2021). Uniform asymptotics for the finite-time ruin probability of a generalized bidimensional risk model with Brownian perturbations. Stochastics: An International Journal of Probability and Stochastic Processes, 93(1), 56-71.

Cheng, D., \& Yu, C. (2019). Uniform asymptotics for the ruin probabilities in a bidimensional renewal risk model with strongly subexponential claims. Stochastics: An International Journal of Probability and Stochastic Processes, 91(5), 643-656.

D’Amico, G., Gismondi, F., Janssen, J., \& Manca, R. (2015). Discrete time homogeneous Markov processes for the study of the basic risk processes. Methodology and Computing in Applied Probability, 17(4), 983-998.

Dermitzakis, V., \& Politis, K. (2011). Asymptotics for the moments of the time to ruin for the compound Poisson model perturbed by diffusion. Methodology and Computing in Applied Probability, 13(4), 749761.

Embrechts, P., Kluppelberg, C., \& Mikosch, T. (1997). Modelling extremal events for insurance and finance. Springer, New York.

Embrechts, P., \& Veraverbeke, N. (1982). Estimates for the probability of ruin with special emphasis on the possibility of large claims. Insurance Mathematics and Economics, 1(1), 55-72.

Foss, S., Korshunov, D., \& Zachary, S. (2011). An introduction to heavy-tailed and subexponential distributions. Springer, New York.

Foss, S., Korshunov, D., Palmowski, Z., \& Rolski, T. (2017). Two-dimensional ruin probability for subexponential claim size. Probability and Mathematical Statistics, 37(2), 319-335.

Fu, K.A., Shen, X.M., \& Li, H.J. (2021). Precise large deviations for sums of claim-size vectors in a twodimensional size-dependent renewal risk model. Acta Mathematicae Applicatae Sinica, 37(3), 539-547. 
Gajek, L., \& Rudź, M. (2021). General methods for bounding multidimensional ruin probabilities in regimeswitching models. Stochastics: An International Journal of Probability and Stochastic Processes, 93(5), 764-779.

Hao, X., \& Tang, Q. (2008). Uniform asymptotic estimate for discounted aggregate claims with subexponential tails. Insurance Mathematics and Economics, 43(1), 116-120.

Jordanova, P.K., \& Stehlık, M. (2018). On multivariate modifications of Cramer-Lundberg risk model with constant intensities. Stochastic Analysis and Applications, 36(5), 858-882.

Kim, M., \& Lee, S. (2008). Estimation of a tail index based on minimum density power divergence. Journal of Multivariate Analysis, 99(10), 2453-2471.

Konstantinides, D.G., \& Li, J. (2016). Asymptotic ruin probabilities for a multidimensional renewal risk model with multivariate regularly varying claims. Insurance Mathematics and Economics, 69, 38-44.

Korshunov, D.A. (2002). Large-deviations probabilities for maxima of sums of independent random variables with negative mean and subexponential distribution. Theory of Probability and its Applications, 46(2), 355-366.

Kostyuchenko, Y.V. (2018). On the methodology of satellite data utilization in multi-modeling approach for socio-ecological risks assessment tasks: A problem formulation. International Journal of Mathematical, Engineering and Management Sciences, 3(1), 1-8.

Leipus, R., \& Siaulys, J. (2007). Asymptotic behaviour of the finite-time ruin probability under subexponential claim sizes. Insurance Mathematics and Economics, 40(3), 498-508.

Leipus, R., \& Siaulys, J. (2009). Asymptotic behaviour of the finite-time ruin probability in renewal risk models. Applied Stochastic Models in Business and Industry, 25(3), 309-321.

Li, J. (2015). Asymptotics for large claim reinsurance in a time-dependent renewal risk model. Scandinavian Actuarial Journal, 2015(2), 172-183.

$\mathrm{Li}$, J. (2016). Uniform asymptotics for a multi-dimensional time-dependent risk model with multivariate regularly claims and stochastic return. Insurance Mathematics and Economics, 71, 195-204.

Li, J., \& Yang, H. (2015). Asymptotic ruin probabilities for a bidimensional renewal risk model with constant interest rate and dependent claims. Journal of Mathematical Analysis and Applications, 426(1), 247-266.

Liu, L., Yang, B., \& Long, G. (2007). The finite time ruin probability of a bidimensional risk model with heavy-tailed claims. Math. Theory and Appl, 27(2), 67-71.

Lu, D., \& Zhang, B. (2016). Some asymptotic results of the ruin probabilities in a two - dimensional renewal risk model with some strongly subexponential claims. Statistics and Probability Letters, 114, 20-29.

Psarrakos, G. (2015). On the integrated tail of the deficit in the renewal risk model. Methodology and Computing in Applied Probability, 17(2), 497-513.

Sun, H., Geng, B., \& Wang, S. (2021a). Asymptotic sum-ruin probability for a bidimensional risk model with common shock dependence. Stochastics: An International Journal of Probability and Stochastic Processes, 93(7), 1028-1042.

Sun, H., Geng, B., \& Wang, S. (2021b). Asymptotic sum-ruin probability for a bidimensional renewal risk model with subexponential claims. Communications in Statistics - Theory and Methods, 1-15. DOI: 10.1080/03610926.2021.1944215 (to appear).

Tang, Q. (2004). Asymptotics for the finite time ruin probability in the renewal model with consistent variation. Stochastic Models, 20(3), 281-297.

Toma, A., \& Dedu, S. (2014). Quantitative techniques for financial risk assessment: A comparative approach using different risk measures and estimation methods. Procedia Economics and Finance, 8, 712-719. 
Tang, Q., Su, C., Jiang, T., \& Zhang, J. (2001). Large deviations for heavy-tailed random sums in compound renewal model. Statistics and Probability Letters, 52(1), 91-100.

Veraverbeke, N. (1977). Asymptotic behaviour of Wiener-Hopf factors of a random walk. Stochastic Processes and Their Applications, 5(1), 27-37.

Wang, S., Qian, H., Sun, H., \& Geng, B. (2021). Uniform asymptotics for ruin probabilities of a non standard bidimensional perturbed risk model with subexponential claims. Communications in Statistics - Theory and Methods, 1-16. DOI: 10.1080/03610926.2021.1882496 (to appear). 